\title{
Position-based Data Dissemination to Interested Region in VANETs
}

\author{
Shuoping Wang ${ }^{1}$, Meng $\mathrm{Kuai}^{2}$ and Honghao $\mathrm{Gao}^{3}$ \\ ${ }^{1}$ Zhejiang University City College, Hangzhou P.R. China \\ ${ }^{2}$ University of Alabama, Tuscaloosa, AL, USA \\ ${ }^{3}$ Computing Center, Shanghai University, 200444 Shanghai, P.R. China \\ wangsp@zucc.edu.cn
}

\begin{abstract}
Vehicular Ad-hoc Networks (VANETs) are expected to provide assistance to various applications, such as accident broadcast or emergency notification. Some kinds of applications need data to be delivered in a certain region by geocast, which means disseminating information in a specific area, instead of using a certain IP address. In this paper, we propose a data dissemination protocol for VANETs, which delivers data to all the vehicles in an interested region. We assume each vehicle is equipped with GPS and DSRC devices. Our simulation results show that this data dissemination protocol can contribute to high delivery ratio. Also, it performs well in adaptability during heavy traffic.
\end{abstract}

Keywords: VANETs, data dissemination, Geocast, position-based

\section{Introduction}

A Vehicular Ad-Hoc Network, or VANET is a technology that uses moving vehicles as nodes in a network to create a mobile network [1]. Normally, vehicles communicate with others via Dedicated Short Range Communication (DSRC) and Wireless Access in Vehicular Environment (WAVE). With the widely use of Global Position System (GPS), devices on a vehicle can easily catch and analysis its current position. With advanced communication device, it can share information with other vehicles.

Also, with the development of wireless communication technologies, Intelligent Transport Systems (ITS) have been attracting more and more public attention. Intelligent Transport Systems are advanced applications, which, without embodying intelligence as such, aim to provide innovative services relating to different modes of transport and traffic management and enable various users to be better informed and make safer, more coordinated, and 'smarter' use of transport networks [2]. In ITS, different applications have various communication requirements. Emergency notification requires low delay. Private message deliver needs high secrecy.

Some proposed applications require message to be disseminated in a certain region. Example is when an accident occurs on a road, the involving cars need to send accident message to the coming cars. Anther scenario is that when an emergency vehicle comes, it will notify emergency notification message to all the vehicles on its coming route. Geocast is innovation in VANETs differing from traditional routing method because messages belong to a certain region rather than any vehicles.

In this paper, we propose a position-based data dissemination protocol for messages to be delivered to all vehicles in a certain region. Nodes exchange location information with its neighbors by continuous beacons. At some time, source sends a message, which includes data and destination region information. The destination region is denoted as the coordinators of 
two points constructing a rectangle. If the source is inside the interested region, it will broadcast the message. Otherwise, the message will be forwarded intermediary using modified GPSR forwarding methods. Any receiver outside the region will forward the message to the region using the same method as the source. As long as there is one node in the region receive the message, it will transform the routing method by broadcasting the message to all its neighbors.

We use Simulation of Urban Mobility (SUMO) to simulate vehicular mobility and have implemented this data dissemination protocol in NS-3. The results of evaluation show that this protocol can contribute to fairly high delivery ratio. In addition, it performs well in adaptability when the traffic is very heavy.

The rest of the paper is structured as follows: Section 2 gives some background and related work. Section 3 describes the proposed protocol. Section 4 presents the simulation and the results. Section 5 concludes the paper and future work. Finsih with the acknowledgement.

\section{Related Work}

In VANETs, position information is a significant factor in routing protocols. There are some proposed position-based protocols that use position information to make routing decisions. In [4] and [5], they proposed the classic routing protocol using geographical distance to the destination help make next-hop selection decisions. In these routing protocols, the nodes need to know the exact position of the destination, which is implemented by using a location service.

One of the most efficient position-based routing protocols is GPSR [3]. In this protocol, it uses greedy forwarding, which means a node that wants to forward a message will choose its neighbor that is closest to the destination. Greedy forwarding strategies can run into a situation called local maximum or local optimum, in which the sending vehicle is closer to the destination than all of its neighbors, and the destination is not reachable by one hop [6]. In addition, it has a recovery-mode strategy or perimeter mode, which is used when greedy forwarding is not able to find a neighbor node that is closer to destination to it.

In the latest version of NS-3, it doesn't include implemented geographical routing protocol. But in [6], they have already implemented GPSR in NS-3. Experiment proves that the GPSR module works as expected, since the paths were correctly chosen according to the protocol specifications, both in greedy and recovery mode. In our simulation, we take advantage of the GPSR module implemented in [6] and add some new functions that adapt to geocast requirements.

There are some researchers focusing on keeping useful information to remain at a confined area. In [7], they proposed a scheme to apply epidemic routing within the hovering area and probabilistic flooding outside the area. To avoiding the effect of low traffic density, they used probabilistic flooding to exchange controlled message outside the area. The Gaussian-like function as the best performance is selected and tuned to evaluate the scheme. The simulation shows that in extreme low traffic density, the adaptive scheme has similar behavior as blind flooding, and with increasing the density; the adaptive scheme can reduce the number of messages and keep higher reachability.

In [8], to avoid beacon collision, they proposed a schedule method to enable vehicles broadcast beacons in desynchronized way, based on timing information. The method will adjust the firing time of nodes with the same phase and use multiplicative increase beaconing to handle different periods. The result shows reduced the beacon collision greatly. However, the paper assumed the surrounding network is reliable. 
Some researchers are focusing on using location information to assist communication. In [13], they developed a location-dependent information-sharing scheme. The scheme distributes the replicas along the routes between the main street and the source. Request messages are directed to the main street. The method will cause large traffic on areas with high vehicle density. In addition, more statistics about street traffic is needed. In [14], authors proposed a method to broadcast beacons not in periods and considers the intersection scenarios. The method is based on contention-based forwarding and sets forwarding timer. A node set ordinary rebroadcast timer to forward a message when receiving a message. The intersection-rebroadcast timer is set when the node heads to or leaves the intersection. The method relies on the real time information of the location and there is possible beacon collision when two nodes have the same time.

Some methods are using more specific location information to help making routing decisions. [15] focused on simple beacons for exchanging safety critical information of Intersection Collision Warning System (ICWS) at intersections. Risk situations a categorized in to No-crash, Safe, Attention, and Critical by the severity of the intersection approaches. The simulation results showed that beacon messages relayed by a parking car were more helpful for collision avoidance when the beacon interval was fast enough. However, this relay also generated high false warning rate.

\section{Proposed Protocol}

In this section, we propose a method to disseminate data to an interested region efficiently by using position information. The data dissemination protocol assumes that each vehicle is equipped with a GPS receiver, communication devices that are able to send and receive data with other vehicles. Vehicles exchange packets using short-range wireless interfaces such as IEEE 802.11 [9] and dedicated short-range communication (DSRC) [10].

We need to classify the vehicles into three types: source, uninterested vehicle and interested vehicle. Source is the vehicle that generates the traffic message. Uninterested vehicles are those vehicles outside the interested region. They do not need the traffic information. But they can be used as forwarding nodes. Interested vehicles are inside the interested region. They need the traffic message generated from source. Because vehicles are moving all the time, the classification would change with the mobility of vehicles.

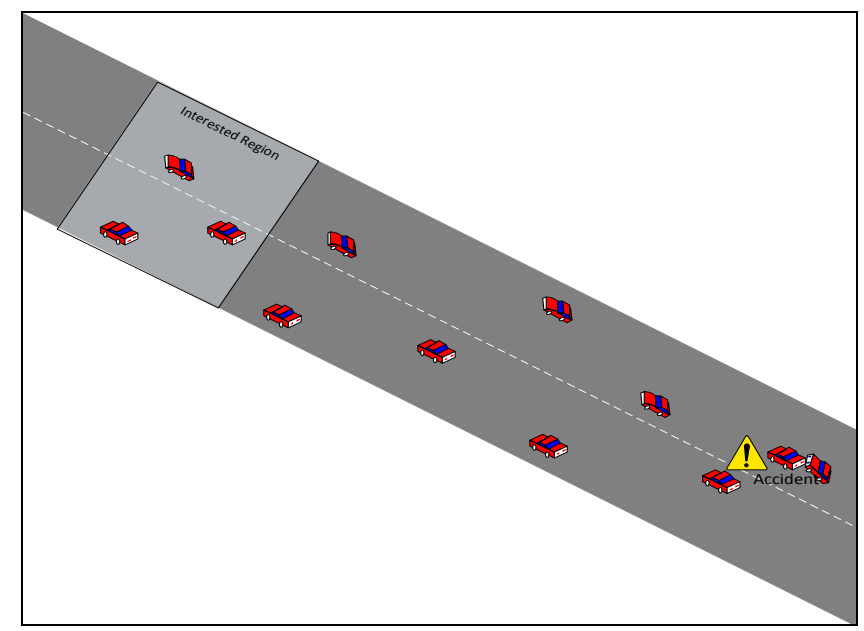

Figure 2. Protocol Scenario 


\section{A. Source}

We regard a source as a static vehicle, a stopped police car for example. A source can generate emergence information or accident notification and configure an interested region in which all vehicles would need the information. The format of message is shown in Table I. Time stands for the time stamp when the message is sent from source. Area means the interested region, which is denoted as the coordinators of two points constructing a rectangle. Data is the actual valid data content, which includes traffic information, such as emergency information or accident notification.

\section{Table I. Data Format of Message}

\begin{tabular}{|l|l|l|}
\hline Time & Area $((\mathrm{x}, \mathrm{y}),(\mathrm{x}, \mathrm{y}))$ & Data \\
\hline
\end{tabular}

\section{B. Neighbor Discovery}

Each vehicle will broadcast Hello message continuously, which only includes its own current position. To mitigate the problem of neighbors generating Hello message at the same time, a random jitter has been used. The jitter is at most equal to half of the interval for sending Hello message. In this scheme, neighbors that happen to send Hello message at the same time that collide will not continue having collisions for subsequent Hello messages.

Each node has a neighbor list, as show in Table II. ID is identification for each node by recognizing its MAC address. Position is saved from the header of Hello message. Time is recorded as the local time of the node when it receives the Hello message. When a vehicle receives a Hello message, it will update its neighbor list: insert new neighbor, update the current neighbor, or delete expired neighbors. We set the expired time as twice of the interval for Hello message. Deleting expired neighbors are also used when forwarding, guaranteeing the neighbor list is the latest.

\section{Table II. Data Format of Neighbor List}

\begin{tabular}{|l|l|l|}
\hline ID & Position $(\mathrm{x}, \mathrm{y})$ & Time \\
\hline
\end{tabular}

\section{Unicast-Forwarding}

Each vehicle outside the interested region has responsibility to forward the message toward to the destination area. When a vehicle find out it is an uninterested vehicle by analyzing the header of message and compare its current position, it will enter unicastforwarding mode.

Traditionally, in GPSR [3], it would choose its neighbor, which is nearest to the destination. But in our scenario, the destination is denoted as a rectangle. So we choose the middle point of this rectangle as the destination to choose the best neighbor.

Another issue is when considering signal attenuation during the transmission, choosing farthest neighbor will contribute to high packet loss ratio. So in this scheme, we reduce the candidates by limiting the distance to $\alpha$, which can be calculated by the equation:

$$
\alpha=\beta-\mu
$$

In equation (1), $\beta$ indicates the transmission range when sending message to the neighbor. $\mu$ is a constant. We set it 20 meters after comparing other values. After 
getting the value of $\alpha$, an uninterested vehicle can choose best neighbor with more reliable link as the next hop of the message.

\section{Broadcast-Forwarding}

When a vehicle confirms it is interested vehicle when it receives a message, it will enter broadcast-forwarding mode, in which vehicle will broadcast the message to all its neighbors, and then deliver the message. To mitigate the message flooding, each interested vehicle will broadcast message only once.

\section{Simulation and Results}

In this section, we will present the evaluation of the data dissemination protocol using network simulator NS-3 [11]. To evaluate the performance, we use the mobility trace generated by SUMO [12]. Our traffic scenario is a highway. Cars are generated from one side. When they reach one end of the road, they will come back to keep the number of cars constant.

\section{A. Simulation Setup}

Table III shows the configuration of simulation. For detecting the effect of traffic density on protocol, we choose several cases by changing vehicle number from 10 to 90. Traffic scenario is chosen as a piece of highway, which is 1000 meters. Source is located on one side of road. Interested area is located as the center of road, with size of $200 \mathrm{~m} \times 200 \mathrm{~m}$.

\section{Table III. Simulation Configuration}

\begin{tabular}{|c|c|}
\hline Parameter & Value \\
\hline Vehicle Number & $10 \sim 90$ \\
\hline Topology Size & $1000 \mathrm{~m}$ \\
\hline Interested Area Size & $200 \mathrm{~m} \times 200 \mathrm{~m}$ \\
\hline Acceleration & $1 \mathrm{~m}^{2} / \mathrm{s}$ \\
\hline Deceleration & $5 \mathrm{~m}^{2} / \mathrm{s}$ \\
\hline Speed Limit & $10 \mathrm{~m} / \mathrm{s}$ \\
\hline Lane Number & 2,3 \\
\hline Minimal Gap & $2 \mathrm{~m}$ \\
\hline Transmission Range & $110 \mathrm{~m}$ \\
\hline Hello Interval & $2 \mathrm{~s}$ \\
\hline
\end{tabular}

\section{B. Evaluation Metrics}

To compare our proposed protocol, we implemented first contact and epidemic protocols. In first contact routing, message is forwarded to the first encountered node. In epidemic routing, message is forwarded to each encountered node. Notice that there is only one message copy in first case, but muiltiple copies in later case.

The performance of the data dissemination protocol was evaluated by varying the number of vehicles. The metrics to assess the performance are given as follows.

- Delivery Ratio. This metric is defined as the number of messages that are successfully delivered at any vehicle in an interested region per the number of messages that are sent by the source. The delivery ratio shows the ability of data dissemination protocol to successfully disseminate data to an interested region. 
- Delay. This metric is defined as the average delay for each message that is successfully delivered in the interested region. The average delay characterizes the latency that the data dissemination protocol creates.

- Hops. This metric is defined as the average number of hops for each message that is successfully delivered in the interested region.

- Number of Forward. This metric is defined as the total number of forward for each message that is successfully delivered in the interested region. It includes the unicast forward and broadcast forward.

\section{Results Analysis}

We evaluate the data dissemination protocol in terms of the above four metrics.

In Figure 2, $\mathrm{X}$-axis denotes the different cases according to different number of vehicles. Y-axis represents the message delivery ratio. The increased curve comes from the factor that traffic density affects the ability of data dissemination protocol. But after a certain number of vehicles, this kind of affect have been become weaken. Notice that when the number of vehicles becomes 50 and more, the delivery ratio is close to one, which means all the messages generated by the source have been delivered in the interested region. It proves the data dissemination protocol has a strong ability to deliver data to a certain region. Epidemic routing has the largest delivery ratio, which is because it forward message to any neighbor nodes. First contact has the smallest delivery ratio, which is due to a certain part of message get lost during the dissemination process.

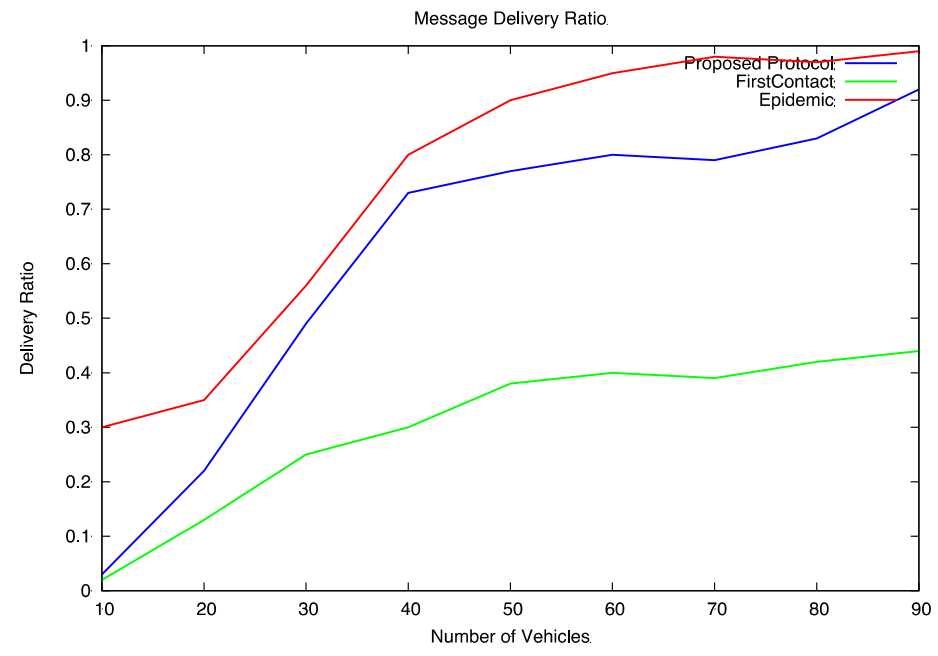

Figure 2. Results of Evaluation - Delivery Ratio

Figure 3 shows that this data dissemination protocol can lead to small delay even thought the vehicles in traffic are overwhelmed. Before the point 20, the curve increases very fast. This is because in sparse traffic, the condition of link is good enough to guarantee high communication quality. Even though the delivery ratio is not high, successful delivers have low average delay. After point 20, the increase is not notable due to it reaches a potential maximum value. 


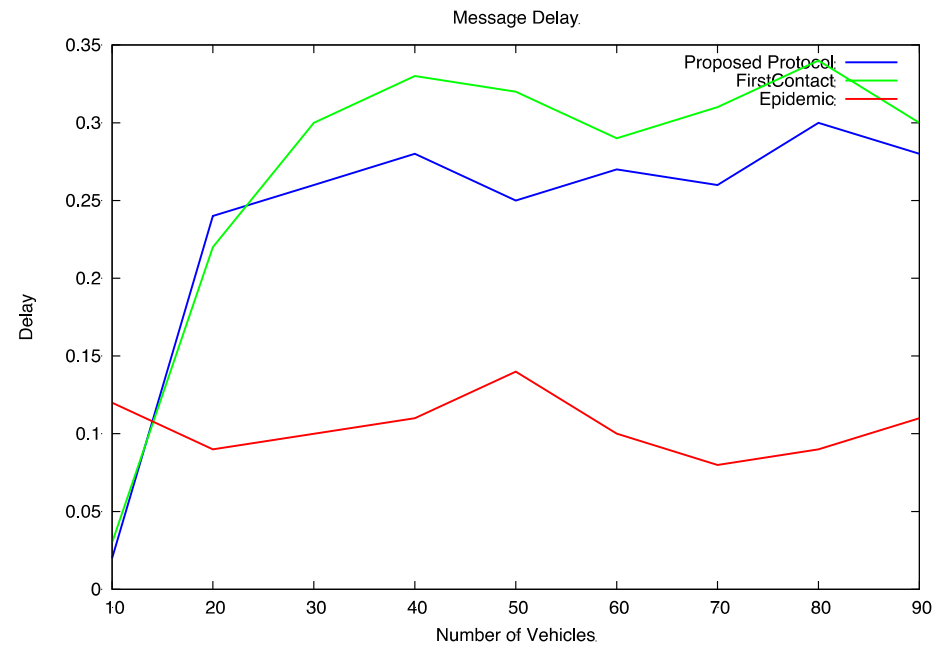

Figure 3. Results of Evaluation - Delay

We notice from Figure 4 that the hops for each successful delivery are changed with the increase of vehicle numbers. The rising segment of the curve corresponds to the case that in sparse traffic, count of hops increases with the increase of vehicles' number. But in dense traffic, hops' number will drop down with the increase of vehicles' number.

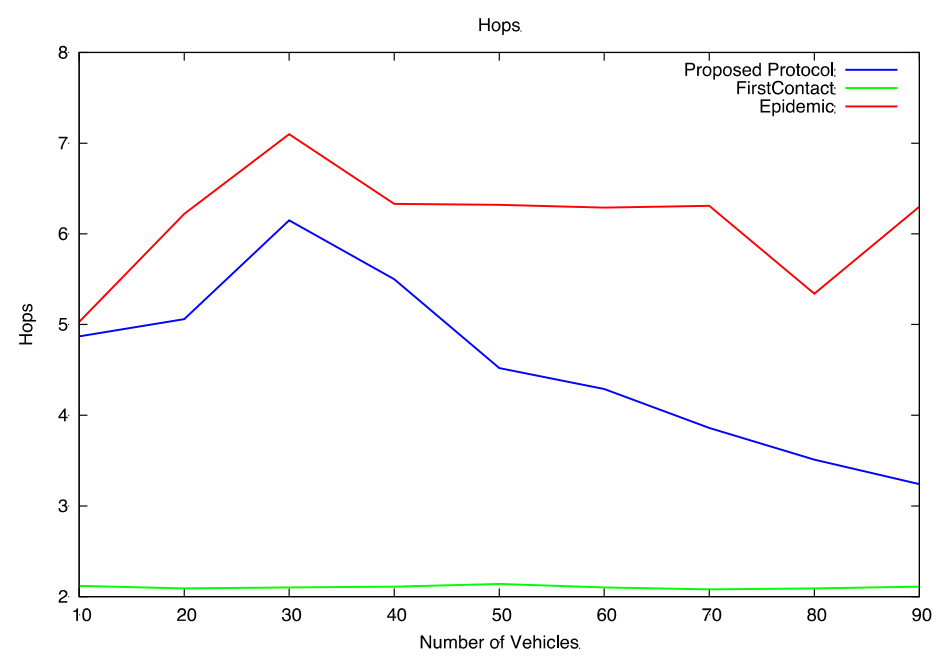

Figure 4. Results of Evaluation - Hops

Figure 5 describes the relationship between average number of forwards for successful delivers and the number of vehicles for different traffic cases. We observe an obvious increasing curve. This is because more dense traffic will contribute to more vehicles in the interested region, which will lead to more forwards in the mode of broadcast. 


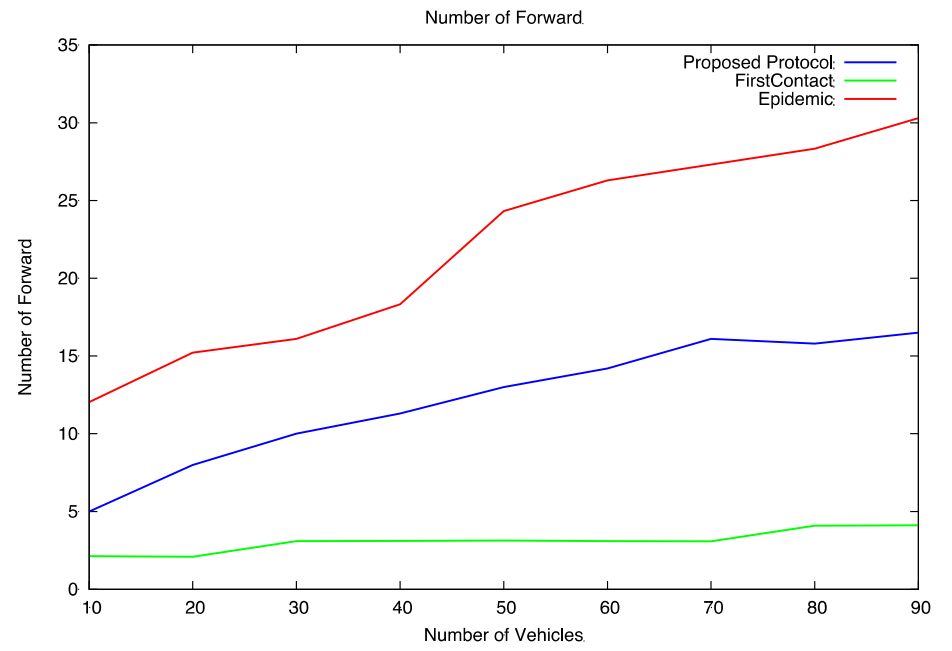

Figure 5. Results of Evaluation - Number of Forward

\section{Conclusions and Future Work}

This paper has presented a data dissemination protocol, which uses geocast routing method to deliver message to all vehicles in a certain region. To adapt characteristics of geocast and improve delivery ratio of message, it modified the scheme of GPSR. Simulation results showed it was able to reach close to 100 percent delivery ratio when there exist enough vehicles. In the future work, we should use urban road scenario for simulation. Also, we need to compare this protocol with other geocast protocol.

\section{Acknowledgements}

This work has been supported by Hangzhou Key Laboratory for IoT Technology \& Application and Young University Teachers Training Plan of Shanghai Municipality under Grant No. ZZSD13008.

\section{References}

[1] http://en.wikipedia.org/wiki/Vehicular_ad-hoc_network.

[2] http://en.wikipedia.org/wiki/Intelligent_transportation_system.

[3] B. Karp and H.-T. Kung, "GPSR: Greedy perimeter stateless routing for wireless networks", Proceedings of the 6th annual international conference on Mobile computing and networking.

[4] H. Takagi and L. Kleinrock, "Optimal transmission ranges for randomly distributed packet radio terminals", Communications, IEEE Transactions on, vol. 32.3, (1984), pp. 246-257.

[5] G. G. Finn, "Routing and addressing problems in large metropolitan-scale internetworks", No. ISI/RR-87180, UNIVERSITY OF SOUTHERN CALIFORNIA MARINA DEL REY INFORMATION SCIENCES INST, (1987).

[6] A. Fonseca, A. Camões and T. Vazão, "Geographical routing implementation in NS3", Proceedings of the 5th International ICST Conference on Simulation Tools and Techniques, ICST (Institute for Computer Sciences, Social-Informatics and Telecommunications Engineering), (2012).

[7] A. Xeros, M. Lestas, M. Andreou and A. Pitsillides, "Adaptive probabilistic flooding for Information Hovering in VANETs", Vehicular Networking Conference (VNC), IEEE, pp. 239,246, (2010) December 1315.

[8] T. Settawatcharawanit, S. Choochaisri, C. Intanagonwiwat and K. Rojviboonchai, "V-DESYNC: Desynchronization for Beacon Broadcasting on Vehicular Networks", Vehicular Technology Conference (VTC Spring), 2012 IEEE 75th, (2012) May 6-9, pp. 1-5. 
[9] "Institute of Electrical and Electronic Engineers (IEEE)", Wireless LAN Medium Access Control (MAC) and Physical Layer Specifications, [Online] Available: http://standards.ieee.org/getieee802/802.11.html.

[10] "Task 3 Final Report," Identify Intelligent Vehicle Safety Applica- tions Enabled by DSRC, DOT HS 809 859, (2005) March, (last accessed Jul. 2008), [Online], Available: http://www-nrd.nhtsa.dot.gov/pdf/nrd-12/ 1665CAMP3web/index.html.

[11] "Ns-3" http://www.nsnam.org.

[12] "Sumo" http://sumo.sourceforge.net.

[13] J. Okamoto and S. Ishihara, "Distributing location-dependent data in VANETs by guiding data traffic to high vehicle density areas", Vehicular Networking Conference (VNC), 2010 IEEE, (2010) December 13-15, pp. 189-196.

[14] Y. Sung and M. Lee, "Light-Weight Reliable Broadcast Message Delivery for Vehicular Ad-Hoc Networks", Vehicular Technology Conference (VTC Spring), 2012 IEEE 75th, (2012) May 6-9, pp. 1-6.

[15] S. Joerer, M. Segata, B. Bloessl, R. L. Cigno, C. Sommer and F. Dressler, "To crash or not to crash: Estimating its likelihood and potentials of beacon-based IVC systems," in Vehicular Networking Conference (VNC), 2012 IEEE, (2012), pp. 25-32. 
International Journal of Grid Distribution Computing Vol.7, No.5 (2014) 